\title{
Negative lymph node count is an independent prognostic factor for female patients with node positive breast cancer
}

\author{
Hao $\mathrm{Wu}^{1} \wedge$, Yajing Huang ${ }^{2} \wedge$ \\ ${ }^{1}$ Department of General Surgery, Tongji Hospital, Tongji Medical College, Huazhong University of Science and Technology, Wuhan, China; \\ ${ }^{2}$ Department of Breast and Thyroid Surgery, Tongji Hospital, Tongji Medical College, Huazhong University of Science and Technology, Wuhan, \\ China \\ Contributions: (I) Conception and design: H Wu; (II) Administrative support: Y Huang; (III) Provision of study materials or patients: H Wu; (IV) \\ Collection and assembly of data: H Wu; (V) Data analysis and interpretation: Y Huang; (VI) Manuscript writing: All authors; (VII) Final approval of \\ manuscript: All authors. \\ Correspondence to: Yajing Huang, MD. Department of Breast and Thyroid Surgery, Tongji Hospital, Tongji Medical College, Huazhong University of \\ Science and Technology, Wuhan, China. Email: d201881672@hust.edu.cn.
}

\begin{abstract}
Background: Negative lymph node (NLN) count has been reported to associate with the prognosis of various cancers. This study aims to reveal the prognostic value of NLN count in breast cancer.

Methods: Clinical characteristics of patients were collected from the Surveillance, Epidemiology, and End Results (SEER) database. The X-tile program was used to determine the optimal cutoffs for NLN count. Univariate and multivariate analysis were used to assess the risk factors for breast cancer-specific survival (BCSS).

Results: The X-tile program identified that cutoff value of 2 and 10 could divide the patients into high, middle and low risk subgroups. According to multivariate analysis, patients with NLN count $\leq 1$, over 60 years old, being black, higher tumor grade, higher $\mathrm{T}$ or $\mathrm{N}$ stage, negative hormone receptor, no radiotherapy or no chemotherapy would more likely suffer poor survival outcome. Subgroup analysis showed that NLN count could still predict survival independently.

Conclusions: NLN count is a potentially effective predictor of breast cancer and is a good supplement for N stage and TNM stage. Combining NLN count with other prognostic factors will be a better predictor for the survival of breast cancer patients.
\end{abstract}

Keywords: Negative lymph node (NLN); breast cancer; survival; modified radical mastectomy

Submitted Jun 17, 2020. Accepted for publication Oct 28, 2020.

doi: $10.21037 /$ tcr-20-2351

View this article at: http://dx.doi.org/10.21037/tcr-20-2351

\section{Introduction}

Breast cancer is the most common cancer (excluding skin cancers) and is the second leading cause of cancer death among women $(1,2)$. Axillary lymph node status is important for the staging of breast cancer to evaluate the recurrence, metastasis and prognosis $(3,4)$. According to the current tumor-node-metastasis (TNM) staging system for breast cancer of American Joint Committee on Cancer
(AJCC) and other committees, 6-10 axillary lymph nodes at least should be collected from the breast cancer specimens for accurate histologic examination and proper tumor stage $(5,6)$. The $\mathrm{N}$ stage is evaluated by the number of positive lymph node (PLN); however, each patient is heterogeneous and the prognosis of the patient is limited and inaccurate by only evaluating the node-stage. Therefore, the concept of negative lymph node (NLN) count has been proposed

^ ORCID: Hao Wu, 0000-0003-0700-8147; Yajing Huang, 0000-0001-7876-165X. 
in a variety of cancers as a complement to $\mathrm{N}$ stage, such as colon (7), cervical (8), gastric (9) and esophagus (10) cancer. In fact, NLN count as a predictor has also been reported in breast cancer, but was not primarily described and is a single-center study (11).

To better assess the association between NLN count and breast cancer survival, we used data from the Surveillance, Epidemiology, and End Results (SEER) database, which is a multicenter and large database established by the National Cancer Institute (NCI), to provide support for this study. We present the following article in accordance with the STROBE reporting checklist (available at http://dx.doi. org/10.21037/tcr-20-2351).

\section{Methods}

\section{Patient characteristics}

Clinical characteristics of patients were obtained from the SEER research data (November 2019 submission) and $\mathrm{R}$ version 3.5.1 was used to identify female patients who underwent axillary lymph node dissection (ALND) diagnosed with invasive ductal and lobular (International Classification of Diseases for Oncology code 8522/3) between 2000 and 2013. Patients with no radiotherapy or radiotherapy after surgery were included. Patients were excluded if they were with bilateral breast cancer, with distant metastasis (M1), had one primary cancer but the breast was not the first one, had undefined $\mathrm{T}$ or $\mathrm{N}$ stage, had incomplete estrogen receptor (ER) and progesterone receptor (PR) status, had unknown cause of death or survival months. Year of diagnosis, age, race, tumor grade, AJCC T stage, AJCC N stage, ER and PR status, radiotherapy and chemotherapy were assessed. As patients with no PLN account for about $2 / 3$ of the total data, we selected patients with node positive for analysis to prevent the bias on the cutoffs of NLN. T0 stage was eliminated because there was only one patient in this group. The study was conducted in accordance with the Declaration of Helsinki (as revised in 2013). Ethical approval was not required because we have signed the SEER research data agreement to access SEER information.

\section{Statistical analysis}

The X-tile program (http://www.tissuearray.org/rimmlab/) was used to determine the cutoffs for NLN count with the minimum $\mathrm{P}$ values and maximum $\mathrm{Chi}^{2}$ test (12). The Kruskal-Wallis test was performed to compare continuous variables. Categorical variables were compared by using the Pearson test. The Kaplan-Meier analysis was used to calculate the survival rate, which were analyzed by Logrank test. The survival curves were plotted by GraphPad Prism version 6.0. The Cox proportional hazard regression model was used to identify the independent prognostic factors for survival. Data analysis was performed using SPSS version 22.0. Difference with $P<0.05$ was considered to be statistically significant.

\section{Results}

\section{Patient characteristics}

A total of 6,230 patients with lymph node positive breast cancer after modified radical mastectomy were included in this analysis, including 492 black, 5,259 white and 479 other race. The median ages of patients was 58 years (range, 21-98 years). The median follow up time was 97 months (0-203 months). The mean number of regional lymph nodes examined was 15 (range, 1-90), the mean number of positive nodes was 5 (range, 1-79) and the median NLN count was 10 (range, $0-77$ ).

\section{The optimal cutoffs for NLN count}

NLN count was treated as continuous variable and then it was validated as an independent prognostic factor by univariate Cox analysis [hazard ratio (HR), 0.926; $95 \%$ CI, 0.918-0.934, $\mathrm{P}<0.001]$. Then, the $\mathrm{X}$-tile program was used to determine the optimal cutoffs for NLN count. As a result, cutoff value of 2 and 10 could divide the patients into high, middle and low risk subgroups (Figure 1), of which the 5 -year breast cancer-specific survival (BCSS) rate were $64.6 \%, 83.0 \%$ and $91.8 \%$, respectively $\left(\chi^{2}=426.0, \mathrm{P}<0.001\right)$. Clinical characteristics of patients in different NLN count subgroups were compared with each other. As shown in Table 1, the age, year of diagnosis, tumor grade, AJCC T stage, AJCC N stage, ER and PR status and radiotherapy were significantly different among patients with different NLN counts. While in these subgroups, the proportion of patients with the same race or chemotherapy were basically analogous.

\section{Univariate analysis of BCSS}

According to univariate analysis in Table 2, patients with NLN count $\leq 1$, over 60 years old, being black, higher tumor grade, higher $\mathrm{T}$ or $\mathrm{N}$ stage, negative hormone receptor 

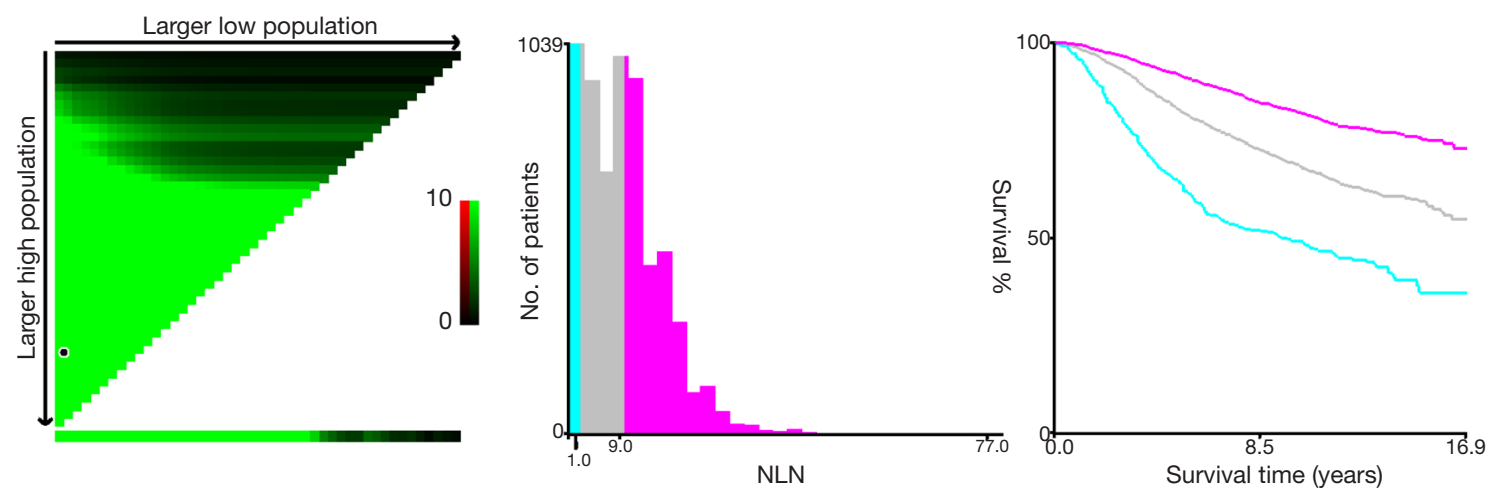

Figure 1 The cutoffs for negative lymph node count was determined by the X-tile program based on 5 -year breast cancer-specific survival $\left(\chi^{2}=425.7, \mathrm{P}<0.001\right)$.

or no radiotherapy would more likely suffer poor survival outcome. Year of diagnosis was associated with significant differences in 5-year BCSS rate, although the correlation is less obvious than other factors $\left(\chi^{2}=3.9, \mathrm{P}=0.048\right)$. Both $\mathrm{ER}$ and $\mathrm{PR}$ positive was associated with higher odds of survival compared with ER or PR positive or ER and PR negative $\left(\chi^{2}=207.4, \mathrm{P}<0.001\right)$. However, according to the data, chemotherapy had no significant effect on 5 -year BCSS rate, which was clinically inconsistent $\left(\chi^{2}=2.6, \mathrm{P}=0.106\right)$.

\section{Multivariate analysis of BCSS}

As shown in Table 2, women with higher NLN count had a lower hazard of death than other subgroups (HR, $0.431 ; 95 \%$ CI, 0.369-0.503, $\mathrm{P}<0.001)$. The white race had a better survival than black race (HR, 0.759; $95 \% \mathrm{CI}$, 0.641-0.898, $\mathrm{P}=0.003)$. Tumor grade, AJCC T or N stage, $\mathrm{ER}$ and $\mathrm{PR}$ status and radiotherapy were also independently prognostic factors $(\mathrm{P}<0.001)$. However, year of diagnosis had no connection with hazard of death, after controlling for other demographic and tumor characteristics. Based on clinical experience, chemotherapy was included in the multivariate analysis and it was found that patients who received chemotherapy would get a better survival (HR, 0.838; 95\% CI, 0.740-0.948, $\mathrm{P}=0.005$ ).

\section{Subgroup analysis of NLN count by Kaplan-Meier survival analysis}

To further analyze the prognostic value of NLN count, different subgroups were ulteriorly divided by NLN count. Figure $2 A$ showed that patients with NLN count $\leq 1$ and older age suffered the highest risk of death. In race subgroup, the curve of black patients with NLN count $\leq 1$ showed the most significant downward trend (Figure 2B). When patients were stratified based on tumor grade, higher NLN count still demonstrated higher survival (Figure 2C). Kaplan-Meier survival analysis of patients stratified by NLN count and AJCC T stage revealed that lower number of NLN and higher T stage resulted in a significantly worse breast cancer BCSS (Figure 2D). Likewise, AJCC N stage subgroup could demonstrate the same result as $\mathrm{T}$ stage (Figure 2E). Hormone receptor-negative breast cancer patients with NLN count $\leq 1$ had the greatest reduction in survival, even compared with other subgroups (Figure $2 F$ ). Subgroups based on whether or not receiving radiotherapy or chemotherapy stratified by the cutoffs of NLN count also showed significant survival differences (Figure 2G,H).

\section{Discussion}

The TNM staging system is routinely used to evaluate survival outcome in patients, in which $\mathrm{N}$ stage represents the number of lymph nodes found to contain metastases, namely PLNs. However, the removed axillary lymph nodes consist of PLNs and NLNs and the number of PLNs is often affected by many facts such as neoadjuvant therapy, so it is not accurate to estimate the prognosis only by the number of PLNs. Recently, several studies have demonstrated that a high NLN count is associated with an improved survival. Mo et al. found that in esophageal cancer a better overall survival (OS) was observed with increasing number of NLNs and NLN count was an independent prognostic factor (13). Likewise, Hao et al. concluded that the high NLN had improved BCSS and OS compared to low NLN and histone modifiers were the most significant 
Table 1 Clinical characteristics of patients in different NLN count subgroups

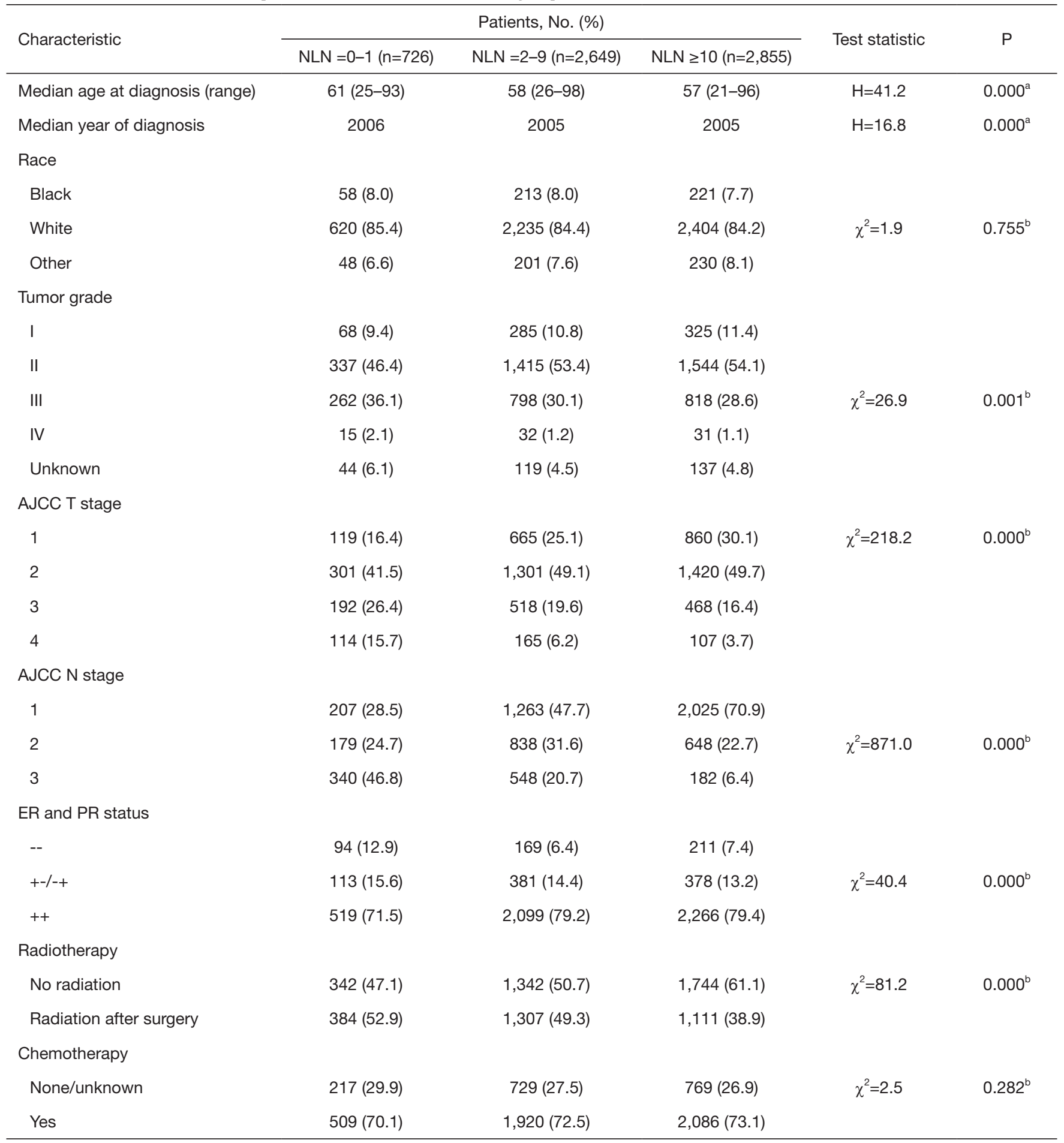

${ }^{a}$, determined by use of the Kruskal-Wallis test. ${ }^{b}$, determined by use of the Pearson test. NLN, negative lymph node; ER, estrogen receptor; $\mathrm{PR}$, progesterone receptor. 
Table 2 Univariate and multivariate analysis of survival with regard to all patients

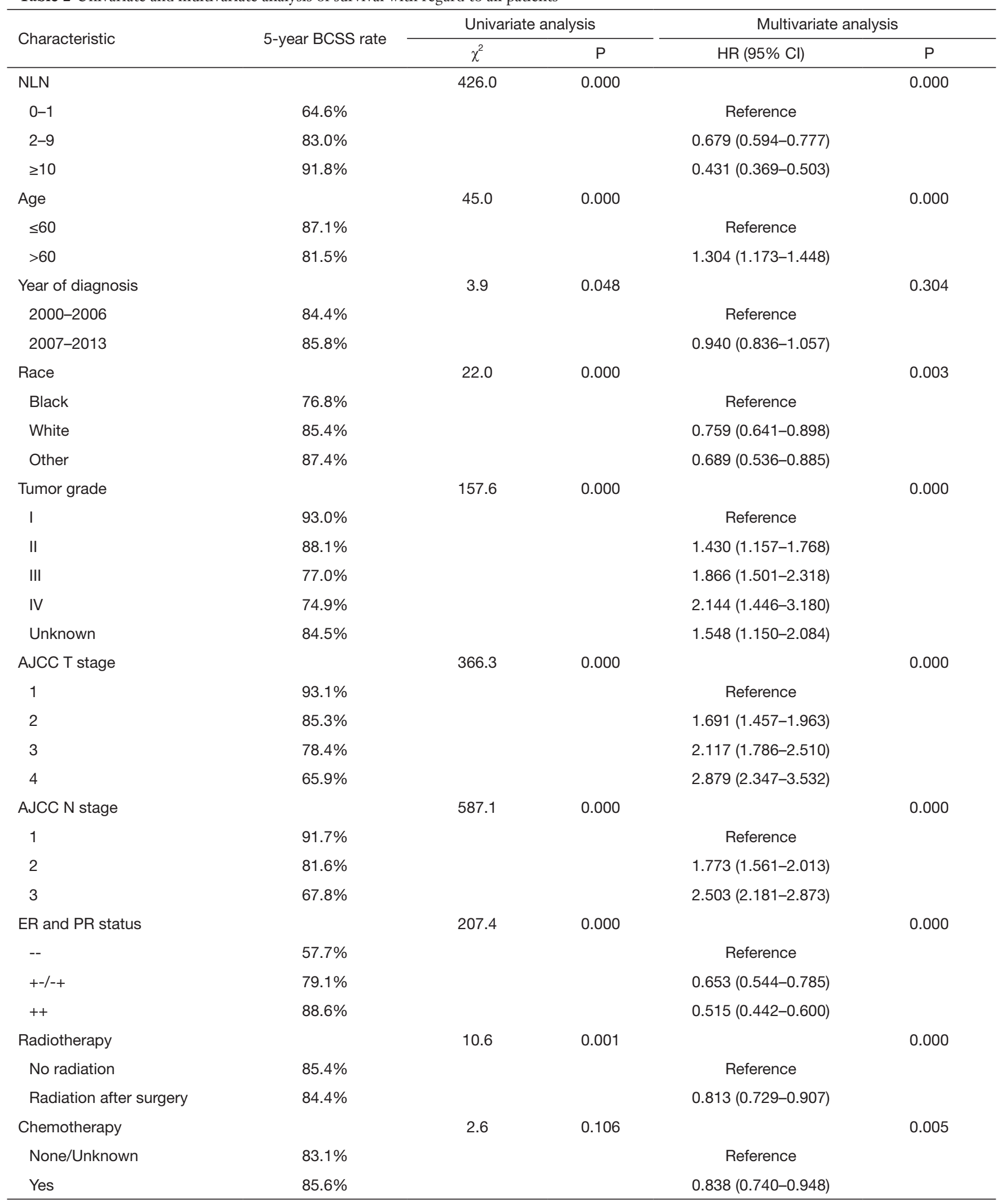

BCSS, breast cancer-specific survival. 

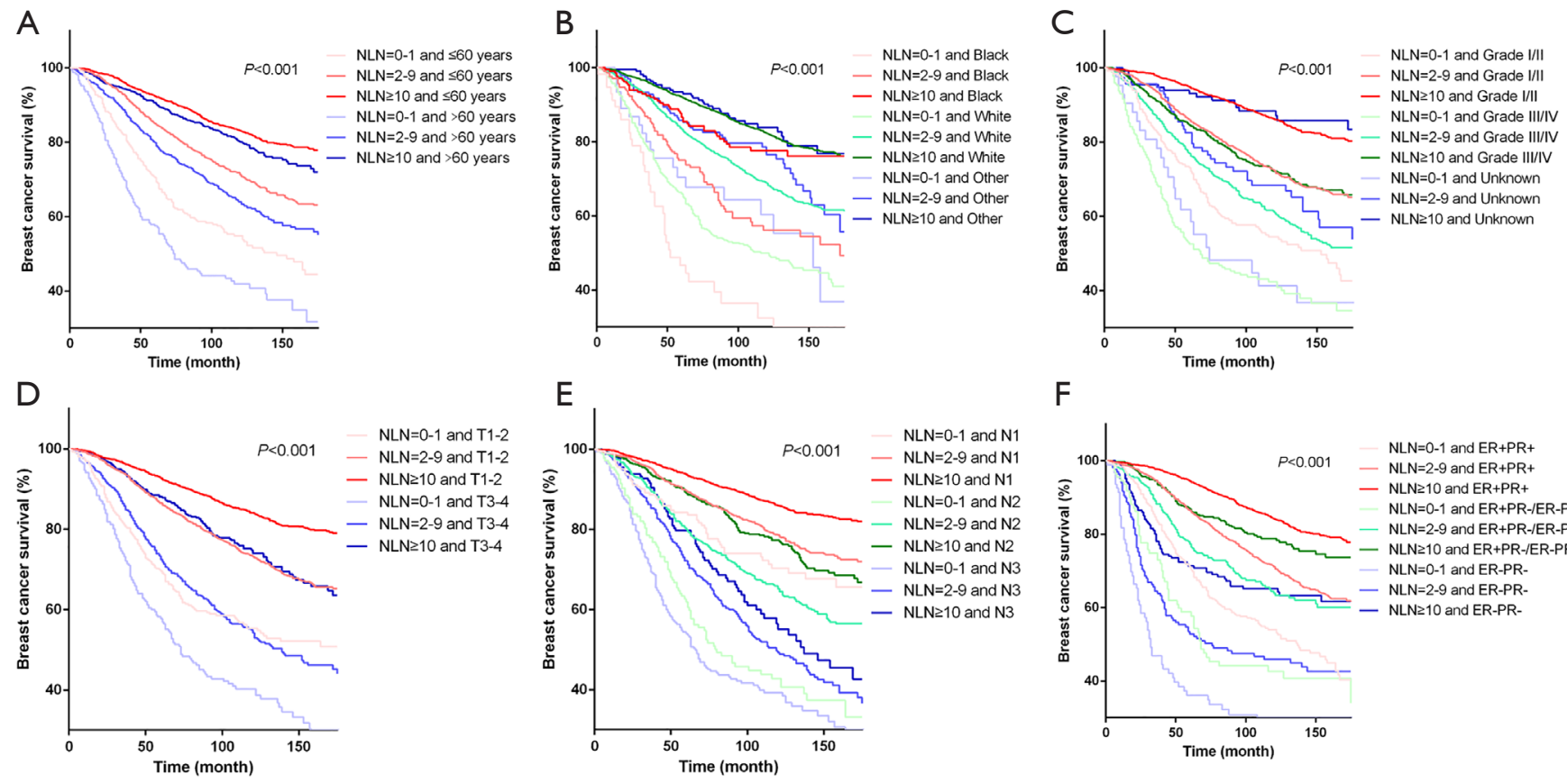

F
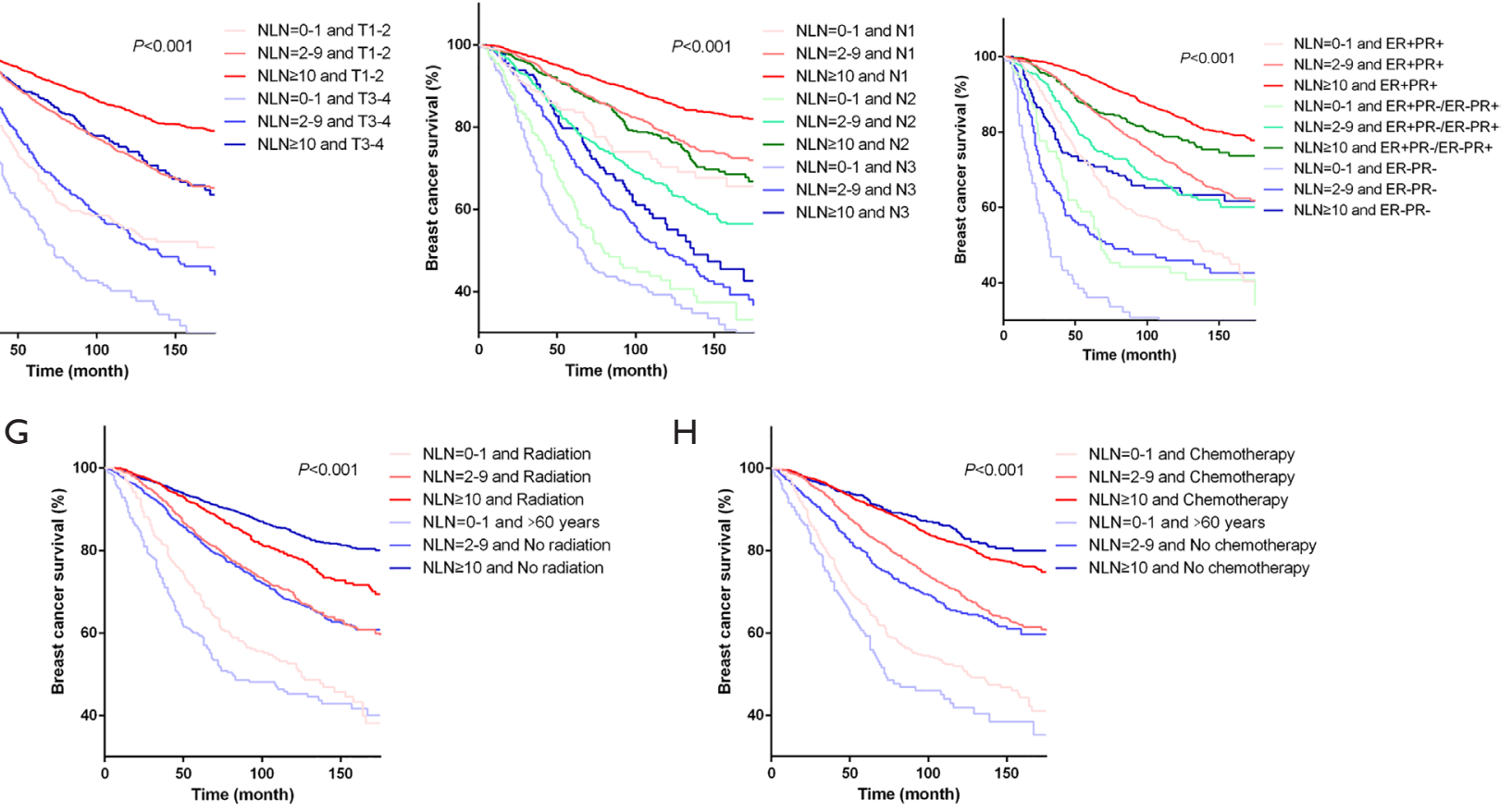

Figure 2 Kaplan-Meier survival analysis of negative lymph node count stratified by (A) age, (B) race, (C) tumor grade, (D) American Joint Committee on Cancer (AJCC) T stage, (E) AJCC N stage, (F) estrogen receptor (ER) and progesterone receptor (PR) status, (G) radiotherapy and $(\mathrm{H})$ chemotherapy.

different biological processes between the high and low NLN group through transcriptomic analysis (14).

In this study, we screened female patients with node positive breast cancer after modified radical mastectomy from the SEER database, and found that NLN count can be a strong predictor for these patients. Additionally, age, race, tumor grade, AJCC T or N stage, radiotherapy and chemotherapy also have association with the survival of breast cancer patients. Even when patients were stratified based on the above subgroups, reduction in NLN count was still strongly associated with lower 5-year BCSS rate. We found that in multivariate analysis chemotherapy was associated with 5-year BCSS rate but not in univariate analysis, which might because the SEER database lacks specific descriptions of chemotherapy regimens and only provides information on whether patients have received chemotherapy. As a result, factors such as patient preferences, physician recommendations, comorbidities, and proximity to treatment providers might lead to bias.

At present, the mechanism by which NLN is associated with survival is unclear, but there are some conjectures. Firstly, the recurrence and metastasis of breast cancer may be achieved by the remaining PLNs, especially those with micrometastases, which is difficult to find during operation $(15,16)$. The more NLNs removed, the fewer lymph nodes with micrometastases is, thus improving survival. 
Secondly, more NLNs means that more total lymph nodes are examined, and the $\mathrm{N}$ stage will be more reliable for the assessment of survival. On the other hand, it reflects the skill of surgeon and the success of the operation. Thirdly, NLN may reflect the response of lymph nodes to the tumor, and the high NLN count may indicate fewer circulating tumor cells, which has an independent effect on survival (17).

With the improvement of breast cancer surgical treatments in recent years, sentinel lymph node biopsy (SLNB) is more and more widely used as the preferred method for axillary lymph node staging of breast cancer patients with clinically negative axillary lymph nodes to replace ALND (18-20). However, SLNB could not obtain enough NLN count, which, according to our findings, may lead to missing a subset of patients with micrometastases and misdiagnosis of breast cancer stage.

In conclusion, our study still suggests that NLN count is a potentially effective predictor of breast cancer and is a good supplement for $\mathrm{N}$ stage and TNM stage. Increased NLN retrieval can improve BCSS. NLN count can predict the prognosis of breast cancer more accurately combined with age, race, tumor grade and stage, receptor status, chemotherapy and radiotherapy.

\section{Acknowledgments}

There was no assistance or efforts beyond those of the primary authors.

Funding: None.

\section{Footnote}

Reporting Checklist: The authors have completed the STROBE reporting checklist, available at http://dx.doi. org/10.21037/tcr-20-2351

Conflicts of Interest: Both authors have completed the ICMJE uniform disclosure form (available at http://dx.doi. org/10.21037/tcr-20-2351). The authors have no conflicts of interest to declare.

Ethical Statement: The authors are accountable for all aspects of the work in ensuring that questions related to the accuracy or integrity of any part of the work are appropriately investigated and resolved. The study was conducted in accordance with the Declaration of Helsinki (as revised in 2013). Ethical approval was not required because we have signed the SEER research data agreement to access
SEER information.

Open Access Statement: This is an Open Access article distributed in accordance with the Creative Commons Attribution-NonCommercial-NoDerivs 4.0 International License (CC BY-NC-ND 4.0), which permits the noncommercial replication and distribution of the article with the strict proviso that no changes or edits are made and the original work is properly cited (including links to both the formal publication through the relevant DOI and the license). See: https://creativecommons.org/licenses/by-nc-nd/4.0/.

\section{References}

1. DeSantis CE, Ma J, Gaudet MM, et al. Breast cancer statistics, 2019. CA Cancer J Clin 2019;69:438-51.

2. Siegel RL, Miller KD, Jemal A. Cancer statistics, 2020. CA Cancer J Clin 2020;70:7-30.

3. Brooks $\mathrm{AD}$. When it comes to breast cancer staging, lymph node status is king. J Surg Res 2010;164:67-8.

4. Rose BS, Jiang W, Punglia RS. Effect of lymph node metastasis size on breast cancer-specific and overall survival in women with node-positive breast cancer. Breast Cancer Res Treat 2015;152:209-16.

5. Singletary SE, Allred C, Ashley P, et al. Revision of the American Joint Committee on Cancer staging system for breast cancer. J Clin Oncol 2002;20:3628-36.

6. The Steering Committee on Clinical Practice Guidelines for the Care and Treatment of Breast Cancer. CMAJ 1998;158 Suppl 3:S1-2.

7. Johnson PM, Porter GA, Ricciardi R, et al. Increasing negative lymph node count is independently associated with improved long-term survival in stage IIIB and IIIC colon cancer. J Clin Oncol 2006;24:3570-5.

8. Chen Y, Zhang L, Tian J, et al. Combining the negative lymph nodes count with the ratio of positive and removed lymph nodes can better predict the postoperative survival in cervical cancer patients. Cancer Cell Int 2013;13:6.

9. Deng J, Liang H, Wang D, et al. Enhancement the prediction of postoperative survival in gastric cancer by combining the negative lymph node count with ratio between positive and examined lymph nodes. Ann Surg Oncol 2010;17:1043-51.

10. Zhu Z, Chen H, Yu W, et al. Number of negative lymph nodes is associated with survival in thoracic esophageal squamous cell carcinoma patients undergoing three-field lymphadenectomy. Ann Surg Oncol 2014;21:2857-63.

11. Yang J, Long Q, $\mathrm{Li} \mathrm{H}$, et al. The value of positive lymph 
nodes ratio combined with negative lymph node count in prediction of breast cancer survival. J Thorac Dis 2017;9:1531-7.

12. Camp RL, Dolled-Filhart M, Rimm DL. X-tile: a new bio-informatics tool for biomarker assessment and outcome-based cut-point optimization. Clin Cancer Res 2004;10:7252-9.

13. Mo J, Chen D, Li C, et al. The Significance of Negative Lymph Nodes in Esophageal Cancer After Curative Resection: A Retrospective Cohort Study. Cancer Manag Res 2020;12:1269-79.

14. Hao Y, Zhang J, Du R, et al. Impact of negative lymph nodes on colon cancer survival and exploring relevant transcriptomics differences through real-world data analyses. Ann Transl Med 2019;7:525.

15. Iqbal J, Ginsburg O, Giannakeas V, et al. The impact of nodal micrometastasis on mortality among women with early-stage breast cancer. Breast Cancer Res Treat 2017;161:103-15.

16. Youssef MMG, Cameron D, Pucher PH, et al. The

Cite this article as: $\mathrm{Wu} \mathrm{H}$, Huang Y. Negative lymph node count is an independent prognostic factor for female patients with node positive breast cancer. Transl Cancer Res 2020;9(12):7450-7457. doi: 10.21037/tcr-20-2351 significance of sentinel lymph node micrometastasis in breast cancer: Comparing outcomes with and without axillary clearance. Breast 2016;30:101-4.

17. Vinh-Hung V, Burzykowski T, Cserni G, et al. Functional form of the effect of the numbers of axillary nodes on survival in early breast cancer. Int J Oncol 2003;22:697-704.

18. Krag DN, Weaver DL, Alex JC, et al. Surgical resection and radiolocalization of the sentinel lymph node in breast cancer using a gamma probe. Surg Oncol 1993;2:335-9; discussion 340.

19. Giuliano AE, Kirgan DM, Guenther JM, et al. Lymphatic mapping and sentinel lymphadenectomy for breast cancer. Ann Surg 1994;220:391-8; discussion 398-401.

20. Krag DN, Anderson SJ, Julian TB, et al. Sentinel-lymphnode resection compared with conventional axillarylymph-node dissection in clinically node-negative patients with breast cancer: overall survival findings from the NSABP B-32 randomised phase 3 trial. Lancet Oncol 2010;11:927-33. 